\title{
A survey on Christoffel-Darboux type identities of Legendre, Laguerre and Hermite polynomials
}

\author{
Asghar Arzhang ${ }^{1}$
}

Received: 1 February 2015 / Accepted: 2 September 2015/Published online: 16 September 2015

(c) The Author(s) 2015. This article is published with open access at Springerlink.com

\begin{abstract}
In this paper, we construct some new Christoffel-Darboux type identities for Legendre, Laguerre and Hermite polynomials. We obtain these types of identities for the derivatives of these polynomials.
\end{abstract}

Keywords Christoffel-Darboux identity · Cauchy kernel - Legendre polynomials - Laguerre polynomials . Hermite polynomials

Mathematical Subject Classification 33D45 - 33D50 . $45 \mathrm{E} 05$

\section{Introduction}

In [1], we have simplified the fraction

$P_{n}(x, y)=\frac{P_{n}(y)-P_{n}(x)}{y-x}$

in terms of $P_{i}(x), P_{j}(y)$ where $P_{n} \in\left\{T_{n}, U_{n}, V_{n}, W_{n}\right\}$. Also, for every kind of Chebyshev polynomials, we have obtained the expanded form of the fraction

$P_{n, s}(x, y)=\frac{P_{n}^{(s)}(y)-P_{n}^{(s)}(x)}{y-x}, \quad P_{n} \in\left\{T_{n}, U_{n}, V_{n}, W_{n}\right\}$.

in terms of $P_{i}(x), P_{j}(y)$ where $P_{n}^{(s)}(x)$ is the $s$ th derivative of $P_{n}(x)$.

Asghar Arzhang

arzhang@kiau.ac.ir

1 Department of Mathematics, Karaj Branch, Islamic Azad University, Karaj, Iran
In this paper, we expand the fraction (1) where $P_{n}(x)$ is Legendre, Laguerre and Hermite polynomials.

\section{Christoffel-Darboux type identities of Legendre, Laguerre and Hermite polynomials}

Theorem 2.1 Let $\left\{P_{n}(x)\right\}_{n=0}^{\infty}$ be a sequence of orthogonal polynomials with respect to the weight function $w(x)$ on interval $[a, b]$ then

$$
\begin{aligned}
P_{n+1}(x, y) & =\frac{P_{n+1}(y)-P_{n+1}(x)}{y-x}=\sum_{i=0}^{n} \sum_{j=0}^{n-i} A_{i, j}^{n+1} P_{i}(x) P_{j}(y) \\
& =\sum_{i=0}^{n} \sum_{j=0}^{i} A_{n-i, j}^{n+1} P_{n-i}(x) P_{j}(y)
\end{aligned}
$$

where

$$
\begin{aligned}
& A_{i, j}^{n+1}=\frac{1}{\gamma_{i} \gamma_{j}} \sum_{k=i+j+1}^{n+1} \sum_{v=i}^{k-j-1} C_{n+1, k} B_{v, i} B_{k-v-1, j} \\
&=\frac{1}{\gamma_{i} \gamma_{j}} \sum_{k=0}^{n-i-j} \sum_{v=0}^{k} C_{n+1, k+i+j+1} B_{v+i, i} B_{k+j-v, j} \\
& \gamma_{i}=\int_{a}^{b} P_{n}^{2}(x) w(x) \mathrm{d} x
\end{aligned}
$$$$
B_{m, n}=\int_{a}^{b} x^{m} P_{n}(x) w(x) \mathrm{d} x
$$

and $C_{n+1, k}$ is the coefficient of $x^{k}$ in $P_{n+1}(x)$.

Proof $P_{n}(x)$ is orthogonal to every polynomial of degree less than $n$. So, if $i+j>n$ then $A_{i, j}^{n+1}=0$. If $i+j \leq n$ then use orthogonality and expanded form of $P_{n}(x)$ to obtain the result. 
Corollary 2.1 If the interval $[a, b]$ is symmetric about the origin and $P_{n}(-x)=(-1)^{n} P_{n}(x)$ then for even $n+i+j$, $A_{i, j}^{n}=0$.

If the linearization formula of $P_{n}(x)$ is available then we can compute $A_{i, j}$ coefficients in Eq. (3) by using one sum instead of using double sum in Eq. (4).

\section{Christoffel-Darboux type identities of Hermite polynomials}

Theorem 3.1 Let $H_{n}(x)$ be Hermite polynomial of degree $n$ then

$H_{n}(x, y)=\frac{H_{n}(y)-H_{n}(x)}{y-x}=\sum_{i=0}^{n-1} \sum_{j=0}^{n-i-1} A_{i, j}^{n} H_{i}(x) H_{j}(y)$,

where

$$
\begin{aligned}
A_{i, j}^{n}= & \left(\frac{1-(-1)^{i+j+n}}{2}\right) \\
& \times\left\{\frac{1}{2^{i} i !} \sum_{k=0}^{j}(-1)^{\left(\frac{3 n-3 j+6 k+i+1}{2}\right)} \frac{2^{\left(\frac{n-j+i-1}{2}\right)}}{k !}\left(\begin{array}{c}
n \\
j-k
\end{array}\right)\right. \\
& \times \Gamma\left(\frac{n-j+i+2 k+1}{2}\right)+\frac{1}{2^{j} j !} \sum_{k=0}^{i}(-1)^{\left(\frac{3 n-3 i+6 k+j+1}{2}\right)} \\
& \left.\times \frac{2^{\left(\frac{n-i+j-1}{2}\right)}}{k !}\left(\begin{array}{c}
n \\
i-k
\end{array}\right) \times \Gamma\left(\frac{n-i+j+2 k+1}{2}\right)\right\} .
\end{aligned}
$$

Proof First, we prove that

$$
\begin{aligned}
H_{m, n}(x, y)= & P . V . \int_{-\infty}^{\infty} \int_{-\infty}^{\infty} \frac{H_{m}(x) H_{n}(y) e^{-x^{2}} e^{-y^{2}}}{y-x} d y d x \\
= & 2^{\frac{m+n-1}{2}}(-1)^{n+1} \sin \left(\frac{m+n}{2} \pi\right) \Gamma\left(\frac{m+n+1}{2}\right) \pi \\
& m, n=0,1,2, \ldots
\end{aligned}
$$

From [5], use the Hilbert transform of $H_{n}(y) e^{-y^{2}}$ to obtain

P.V. $\int_{-\infty}^{\infty} \frac{H_{n}(y) e^{-y^{2}}}{y-x} \mathrm{~d} y=(2 \pi)^{n+1} \sqrt{\pi}(-1)^{n+1}$

$$
\times \int_{0}^{\infty} f^{n} e^{-\pi^{2} f^{2}} \sin \left(2 \pi f x+\frac{n \pi}{2}\right) \mathrm{d} f
$$

So

$$
\begin{aligned}
H_{m, n}(x, y)= & P . V \cdot \int_{-\infty}^{\infty} \int_{-\infty}^{\infty} \frac{H_{m}(x) H_{n}(y) e^{-x^{2}} e^{-y^{2}}}{y-x} \mathrm{~d} y \mathrm{~d} x \\
= & (2 \pi)^{n+1} \sqrt{\pi}(-1)^{n+1} \int_{0}^{\infty} \int_{-\infty}^{\infty} f^{n} H_{m}(x) e^{-x^{2}} e^{-\pi^{2} f^{2}} \\
& \times \sin \left(2 \pi f x+\frac{n \pi}{2}\right) \mathrm{d} x \mathrm{~d} f .
\end{aligned}
$$

On the other hand, we have

$$
\begin{aligned}
& \int_{-\infty}^{\infty} e^{-x^{2}} H_{m}(x) \sin \left(2 \pi f x+\frac{n \pi}{2}\right) \mathrm{d} x \\
& = \begin{cases}0, & m+n \text { is even } \\
2 \int_{0}^{\infty} e^{-x^{2}} H_{m}(x) \sin \left(2 \pi f x+\frac{n \pi}{2}\right) \mathrm{d} x, & m+n \text { is odd }\end{cases}
\end{aligned}
$$

So, if $m+n$ is even then $H_{m, n}(x, y)=0$. If $m+n$ is odd, then use relation (8) and integration by parts and Rodrigue's formula of Hermite polynomials to obtain

$$
\begin{aligned}
& \int_{0}^{\infty} e^{-x^{2}} H_{m}(x) \sin \left(2 \pi f x+\frac{n \pi}{2}\right) \mathrm{d} x \\
& \quad=(2 \pi f)^{m} \sin \left(\frac{m+n}{2} \pi\right) \int_{0}^{\infty} e^{-x^{2}} \cos (2 \pi f x) \mathrm{d} x .
\end{aligned}
$$

From the relations (7), (9) by using change of the variable $\pi f=y$, we obtain

$$
\begin{aligned}
H_{m, n}(x, y)= & 2^{m+n+2}(-1)^{n+1} \sqrt{\pi} \sin \left(\frac{m+n}{2} \pi\right) \\
& \times \int_{0}^{\infty} y^{m+n} e^{-y^{2}} \mathrm{~d} y \int_{0}^{\infty} e^{-x^{2}} \cos (2 x y) \mathrm{d} x \\
= & 2^{m+n+2}(-1)^{n+1} \sqrt{\pi} \sin \left(\frac{m+n}{2} \pi\right) \\
& \times \int_{0}^{\infty} y^{m+n} e^{-y^{2}}\left(\frac{\sqrt{\pi}}{2} e^{-y^{2}}\right) \mathrm{d} y \\
= & 2^{\frac{m+n-1}{2}}(-1)^{n+1} \sin \left(\frac{m+n}{2} \pi\right) \Gamma\left(\frac{m+n+1}{2}\right) \pi
\end{aligned}
$$

So, for odd $m+n$, we have

$H_{m, n}(x, y)=2^{\frac{m+n-1}{2}}(-1)^{\frac{m+3 n+1}{2}} \Gamma\left(\frac{m+n+1}{2}\right) \pi$

The famous linearization formula of Hermite polynomials is [2]

$H_{m}(x) H_{n}(x)=2^{m} m ! \sum_{k=0}^{m} \frac{1}{2^{k} k !}\left(\begin{array}{c}n \\ m-k\end{array}\right) H_{n-m+2 k}(x)$,

$$
m \leq n \text {. }
$$

By using the relations (11) and (12), we can obtain $A_{i, j}^{n}$ in relation (5).

Corollary 3.1 The $A_{i, j}^{n}$ coefficients in relation (5) can be computed as follows:

$$
\begin{aligned}
A_{i, j}^{n}= & \frac{n !}{2^{i+j} i ! j ! \pi} \sum_{k=i+j+1}^{n} \sum_{v=i}^{k-j-1}(-1)^{\frac{n-k}{2}}\left(\frac{1+(-1)^{n+k}}{2}\right) \\
& \times\left(\frac{1+(-1)^{v+i}}{2}\right)\left(\frac{1+(-1)^{k+j-v-1}}{2}\right) \\
& \times \frac{2^{k} v !(k-v-1) !}{k !(v-i) !(k-v-j-1) !\left(\frac{n-k}{2}\right) !} \Gamma\left(\frac{v-i+1}{2}\right) \Gamma\left(\frac{k-v-j}{2}\right) .
\end{aligned}
$$


Now, we can obtain Christoffel-Darboux type identities for the derivatives of Hermite polynomials.

Corollary 3.2 Let

$$
\begin{aligned}
H_{n}^{(s)}(x, y)= & \frac{H_{n}^{(s)}(y)-H_{n}^{(s)}(x)}{y-x} \\
= & \sum_{i=0}^{n-s-1} \sum_{j=0}^{n-s-i-1} A_{i, j}^{n, s} H_{i}(x) H_{j}(y), \\
& s=0 \ldots n,
\end{aligned}
$$

where

$$
\begin{aligned}
A_{i, j}^{n, s}= & \frac{2^{s} n !}{(n-s) !}\left(\frac{1-(-1)^{n-s+i+j}}{2}\right) \\
& \times\left\{\frac{1}{2^{i} i !} \sum_{k=0}^{j}(-1)^{\left(\frac{3 n-3 s-3 j+6 k+i+1}{2}\right)} \frac{2^{\left(\frac{n-s-j+i-1}{2}\right)}}{k !}\right. \\
& \times\left(\begin{array}{c}
n-s \\
j-k
\end{array}\right) \Gamma\left(\frac{n-s-j+i+2 k+1}{2}\right) \\
& +\frac{1}{2^{j} j !} \sum_{k=0}^{i}(-1)^{\left(\frac{3 n-3 s-3 i+6 k+j+1}{2}\right)} \frac{2^{\left(\frac{n-s-i+j-1}{2}\right)}}{k !} \\
& \left.\times\left(\begin{array}{c}
n-s \\
i-k
\end{array}\right) \Gamma\left(\frac{n-s-i+j+2 k+1}{2}\right)\right\} .
\end{aligned}
$$

\section{Christoffel-Darboux type identities of Legendre polynomials}

Theorem 4.1 Let $P_{n}(x)$ be Legendre polynomial of degree $n$ then

$P_{n}(x, y)=\frac{P_{n}(y)-P_{n}(x)}{y-x}=\sum_{i=0}^{n-1} \sum_{j=0}^{n-i-1} A_{i, j}^{n} P_{i}(x) P_{j}(y)$,

where

$$
\begin{aligned}
& A_{i, j}^{n}=-\frac{1}{2}(2 i+1)(2 j+1) \\
& \sum_{k=0}^{\min (\mathrm{i}, \mathrm{j})} \frac{1+(-1)^{i+j+n-1}}{i+j+n-2 k+1}\left\{\frac{B_{i, j}^{k}}{i+j-n-2 k}\right. \\
& \left.\quad-\frac{B_{\min (i, j), n}^{k}}{\min (i, j)-\max (i, j)+n-2 k}\right\} \\
& B_{i, j}^{k}=\frac{B_{i-k} B_{k} B_{j-k}}{B_{i+j-k}}\left(\frac{2 i+2 j-4 k+1}{2 i+2 j-2 k+1}\right), \\
& B_{k}=\frac{1 \cdot 3 \cdot 5 \cdots(2 k-1)}{k !}, \quad k=1,2,3, \ldots \\
& B_{0}=1 .
\end{aligned}
$$

Proof Legendre function of the second kind is defined by $Q_{n}(x)=-\frac{1}{2} P . V . \int_{-1}^{1} \frac{P_{n}(y)}{y-x} \mathrm{~d} y$, and

$$
P . V . \int_{-1}^{1} P_{m}(x) Q_{n}(x) \mathrm{d} x=\frac{1+(-1)^{m+n}}{(m-n)(m+n+1)}, \quad m \neq n .
$$

Therefore

$$
\begin{aligned}
A_{i, j}^{n} & =\frac{1}{4}(2 i+1)(2 j+1) \int_{-1}^{1} \int_{-1}^{1} \frac{P_{n}(y)-P_{n}(x)}{y-x} P_{i}(x) P_{j}(y) \mathrm{d} y \mathrm{~d} x \\
& =-\frac{1}{2}(2 i+1)(2 j+1) \int_{-1}^{1} P_{i}(x)\left(P_{j}(x) Q_{n}(x)-P_{n}(x) Q_{j}(x)\right) \mathrm{d} x
\end{aligned}
$$

The following famous linearization formula of Legendre polynomials is Neumann-Adams formula [2]:

$$
\begin{aligned}
& P_{m}(x) P_{n}(x)=\sum_{k=0}^{m} \frac{B_{m-k} B_{k} B_{n-k}}{B_{m+n-k}}\left(\frac{2 m+2 n-4 k+1}{2 m+2 n-2 k+1}\right) \\
& P_{m+n-2 k}(x), \quad m \leq n, \\
& B_{k}=\frac{1 \cdot 3 \cdot 5 \cdot(2 k-1)}{k !}, \quad k=1,2,3, \ldots \\
& B_{0}=1 .
\end{aligned}
$$

Now, use the relations (17), (18) and (19) to obtain the result.

Corollary 4.1 The $A_{i, j}^{n}$ coefficients in relation (15) can be computed as follows:

$$
\begin{aligned}
A_{i, j}^{n}= & \frac{(2 i+1)(2 j+1)}{2^{n+i+j+2}} \sum_{k=i+j+1}^{n} \sum_{v=i}^{k-j-1}(-1)^{\frac{n-k}{2}}\left(\frac{1+(-1)^{n+k}}{2}\right) \\
& \times\left(\frac{1+(-1)^{v+i}}{2}\right)\left(\frac{1+(-1)^{k+j-v-1}}{2}\right) \\
& \times \frac{(n+k) ! v !(k-v-1) !}{k !(v-i) !(k-v-j-1) !\left(\frac{n-k}{2}\right) !\left(\frac{n+k}{2}\right) !} \frac{\Gamma\left(\frac{v-i+1}{2}\right) \Gamma\left(\frac{k-v-j}{2}\right)}{\Gamma\left(\frac{v+i+3}{2}\right) \Gamma\left(\frac{k-v+j+2}{2}\right)}
\end{aligned}
$$

Now, we can obtain Christoffel-Darboux type identities for the derivatives of Legendre polynomials.

From [4], for the case $\gamma=0$, we can derive

$P_{n}^{(s)}(x)=\sum_{k=0}^{n-s} a_{k}^{s} P_{k}(x), \quad s=0 \ldots n$

where

$$
\begin{aligned}
a_{k}^{s}= & \left(\frac{1+(-1)^{n+k+s}}{2}\right) \frac{2 k+1}{2^{s-2}(s-1) !} \frac{(n+k+s-1) !}{(n+k+s+2) !} \\
& \times \frac{\left(\frac{n-k+s}{2}-1\right) !}{\left(\frac{n-k-s}{2}\right) !} \frac{\left(\frac{n+k-s}{2}+1\right) !}{\left(\frac{n+k+s}{2}-1\right) !} .
\end{aligned}
$$




\section{Corollary 4.2}

$$
\begin{gathered}
\frac{P_{n}^{(s)}(y)-P_{n}^{(s)}(x)}{y-x}=\sum_{i=0}^{n-s-1} \sum_{j=0}^{n-s-i-1} A_{i, j}^{n, s} P_{i}(x) P_{j}(y), \\
s=0 \ldots n,
\end{gathered}
$$

where

$$
\begin{aligned}
A_{i, j}^{n, s}= & -\frac{1}{2}(2 i+1)(2 j+1) \sum_{k=i+j+1}^{n-s} \sum_{v=0}^{\min (\mathrm{i}, \mathrm{j})}\left(\frac{1+(-1)^{n+k+s}}{2}\right) \\
& \times \frac{2 k+1}{2^{s-2}(s-1) !} \frac{(n+k+s-1) !}{(n+k+s+2) !} \\
& \times \frac{\left(\frac{n-k+s}{2}-1\right) !}{\left(\frac{n-k-s}{2}\right) !} \frac{\left(\frac{n+k-s}{2}+1\right) !}{\left(\frac{n+k+s}{2}-1\right) !} \frac{1+(-1)^{i+j+k-1}}{i+j+k-2 v+1} \\
& \times\left\{\frac{B_{i, j}^{v}}{i+j-k-2 v}-\frac{B_{\min (\mathrm{i}, \mathrm{j}), \mathrm{k}}^{v}}{\min (i, j)-\max (i, j)+k-2 v}\right\}, \\
B_{i, j}^{v}= & \frac{B_{i-v} B_{v} B_{j-v}}{B_{i+j-v}}\left(\frac{2 i+2 j-4 v+1}{2 i+2 j-2 v+1}\right), \\
B_{v}= & \frac{1 \cdot 3 \cdot 5 \cdots(2 v-1)}{v !}, \quad v=1,2,3, \ldots \\
B_{0}= & 1 .
\end{aligned}
$$

\section{Christoffel-Darboux type identities of Laguerre polynomials}

The famous linearization formula of associated laguerre polynomials is Feldheim formula [6]

$$
\begin{aligned}
L_{m}^{\alpha} L_{n}^{\beta}(x)= & \sum_{k=0}^{m+n} \sum_{v=0}^{k}(-1)^{m+n+k}\left(\begin{array}{l}
k \\
v
\end{array}\right)\left(\begin{array}{c}
m+\alpha \\
n-k+v
\end{array}\right)\left(\begin{array}{c}
n+\beta \\
m-v
\end{array}\right) \\
& \times L_{k}^{\alpha+\beta}(x) .
\end{aligned}
$$

In spite of Hermite and Legendre polynomials, the linearization formula of Laguerre polynomials is presented by double summation. The coefficients $A_{i, j}^{n}$ of Hermite and Legendre polynomials are obtained from (5) and (16) by one summation, and in the following relations, the $A_{i, j}^{n}$ coefficients of Laguerre polynomials are given by double summation.

Corollary 5.1 Let $L_{n}^{m}(x)$ be associated laguerre polynomials of degree $n$ then

$$
\begin{aligned}
L_{n+1}^{m}(x, y) & =\frac{L_{n+1}^{m}(y)-L_{n+1}^{m}(x)}{y-x} \\
& =\sum_{i=0}^{n} \sum_{j=0}^{n-i} A_{i, j}^{n+1} L_{i}^{m}(x) L_{j}^{m}(y),
\end{aligned}
$$

where

$$
\begin{aligned}
& A_{i, j}^{n+1}=\sum_{k=i+j+1}^{n+1} \sum_{v=i}^{k-j-1}(-1)^{i+j+k} \frac{i ! j !}{k !} \\
& \quad \times \frac{(m+v) !(m+k-v-1) !}{(m+i) !(m+j) !}\left(\begin{array}{c}
m+n+1 \\
n-k+1
\end{array}\right)\left(\begin{array}{c}
v \\
i
\end{array}\right)\left(\begin{array}{c}
k-v-1 \\
j
\end{array}\right)
\end{aligned}
$$

The related formula for Laguerre polynomials of degree $n$ is

$$
\begin{aligned}
L_{n+1}(x, y) & =\frac{L_{n+1}(y)-L_{n+1}(x)}{y-x} \\
& =\sum_{i=0}^{n} \sum_{j=0}^{n-i} A_{i, j}^{n+1} L_{i}(x) L_{j}(y),
\end{aligned}
$$

where

$$
A_{i, j}^{n+1}=\sum_{k=i+j+1}^{n+1} \sum_{v=i}^{k-j-1}(-1)^{i+j+k} \frac{\left(\begin{array}{c}
n+1 \\
k
\end{array}\right)\left(\begin{array}{l}
v \\
i
\end{array}\right)\left(\begin{array}{c}
k-v-1 \\
j
\end{array}\right)}{(v+1)\left(\begin{array}{c}
k \\
v+1
\end{array}\right)}
$$

From [3], we have

$\frac{\mathrm{d}^{\mathrm{s}}}{\mathrm{d} x^{s}} L_{n}^{m}(x)=(-1)^{s} \sum_{k=0}^{n-s}\left(\begin{array}{c}n-k-1 \\ s-1\end{array}\right) L_{k}^{m}(x), \quad s=0 \ldots .$.

Let

$L_{n}^{m, s}(x)=\frac{d^{s}}{\mathrm{~d} x^{s}} L_{n}^{m}(x)$,

then

$$
\begin{aligned}
\frac{L_{n}^{m, s}(y)-L_{n}^{m, s}(x)}{y-x}= & \sum_{i=0}^{n-s-1} \sum_{j=0}^{n-s-i-1} A_{i, j}^{n, s} L_{i}^{m}(x) L_{j}^{m}(y), \\
& s=0 \ldots n
\end{aligned}
$$

where

$$
\begin{aligned}
A_{i, j}^{n, s}= & \sum_{k=i+j+1}^{n-s} \sum_{k^{\prime}=i+j+1}^{k} \sum_{v=i}^{k^{\prime}-j-1}(-1)^{i+j+s+k^{\prime}} \\
& \frac{i ! j !}{k^{\prime} !} \frac{(m+v) !\left(m+k^{\prime}-v-1\right) !}{(m+i) !(m+j) !} \\
& \times\left(\begin{array}{c}
n-k-1 \\
s-1
\end{array}\right)\left(\begin{array}{c}
m+k \\
k-k^{\prime}
\end{array}\right)\left(\begin{array}{c}
v \\
i
\end{array}\right)\left(\begin{array}{c}
k^{\prime}-v-1 \\
j
\end{array}\right) .
\end{aligned}
$$




\section{Conclusion}

In this paper, we obtained some new Christoffel-Darboux type identities for Legendre, Laguerre and Hermite polynomials. We also obtained these types of identities for the derivatives of these polynomials. These formulas are good theoretically and the correctness of the obtained formulas are checked by Maple 17, and Some of these formulas are not efficient numerically.

Acknowledgments This work has been funded and supported by Islamic Azad University, Karaj Branch, and the author is thankful to it.

Open Access This article is distributed under the terms of the Creative Commons Attribution 4.0 International License (http://crea tivecommons.org/licenses/by/4.0/), which permits unrestricted use, distribution, and reproduction in any medium, provided you give appropriate credit to the original author(s) and the source, provide a link to the Creative Commons license, and indicate if changes were made.

\section{References}

1. Arzhang, A.: A survey on Christoffel-Darboux-type identities of Chebyshev polynomials. Integral Transforms Spec. Funct. 24(10), 840-849 (2013). doi:10.1080/10652469.2012.762711

2. Andrews, G.E., Roy, R.: Special Functions. Cambridge University Press, Richard Askey, Cambridge (1999)

3. Doha, E.H.: On the connection coefficients and recurrence relations arising from expansions in series of Laguerre polynomials. J. Phys. A 36, 5449-5462 (2003)

4. Karageorghis, A., Phillips, T.N.: On the coefficients of differentiated expansions of ultraspherical polynomials. Appl. Numer. Math. 9, 133-141 (1992)

5. Poularikas, A.D.: The Transforms and Applications Handbook. CRC Press LLC, Boca Raton (2000)

6. Popov, B.S., Srivastava, H.M.: Linearization of a product of two polynomials of different orthogonal systems. Facta Univ. Ser. Math. Inform. 18, 1-8 (2003) 